\title{
Shared Decision Making in a Youth Mental Health Service Design and Research Project: Insights From the Pan-Canadian ACCESS Open Minds Network
}

\author{
Chloé Guinaudie ${ }^{1}$. Chantelle Mireault ${ }^{1} \cdot$ Jimmy Tan $^{1} \cdot$ Yvonne Pelling $^{1} \cdot$ Sara Jalali ${ }^{1}$. Ashok Malla ${ }^{1,2,3}$. \\ Srividya N. Iyer ${ }^{1,2,3}$
}

Published online: 30 September 2020

(c) The Author(s) 2020

\begin{abstract}
Shared decision making (SDM) is the process by which health care providers and patients collaborate to make health care decisions. This collaboration leads to informed decision making and improved outcomes. However, research on SDM specific to the field of youth mental health is scarce. ACCESS Open Minds (ACCESS OM) is a youth mental health research and evaluation project that implemented and evaluated SDM practices within its various activities and operations. The ACCESS OM network spans a diversity of youth mental health settings across Canada, and includes various stakeholders such as youth, family members and carers, clinicians, researchers, and policy makers. The project values all types of knowledge (specifically, experiential, cultural, clinical, and scientific knowledge) as necessary to lead to better health research, care delivery, and outcomes for patients and their communities. Similarly, it acknowledges the lived experience of patients and, family and carers as expertise. Through the integration of SDM practices, ACCESS OM has formulated valuable insights that can be applied to other health problems and settings. This paper, written by youth and family council members, operational staff, and researchers from the project, will share challenges and solutions that arose in the integration of SDM practices within ACCESS OM's knowledge translation strategy, governance structures, clinical contexts, and capacity-building initiatives.
\end{abstract}

\section{Plain Language Summary}

This paper describes how ACCESS Open Minds (ACCESS OM) uses shared decision making (SDM) strategies. ACCESS $\mathrm{OM}$ is a pan-Canadian youth mental health project, which is improving youth mental health services across Canada. Often, health care systems are not set up to prioritize patients' expertise when it comes to decisions about care and services. SDM means that patients, service providers, and other relevant individuals collaborate to make decisions about health care. SDM strategies are important in how ACCESS OM is working to improve youth mental health services. This paper provides

Chloé Guinaudie and Chantelle Mireault co-first authors.

Ashok Malla and Srividya N. Iyer co-last authors.

Digital features To view digital features for this article go to https://doi.org/10.6084/m9.figshare.12722510.

Electronic supplementary material The online version of this article (https://doi.org/10.1007/s40271-020-00444-5) contains supplementary material, which is available to authorized users.

Srividya N. Iyer

srividya.iyer@mcgill.ca

1 ACCESS Open Minds (Pan-Canadian Youth Mental Health Services Research Network), Douglas Mental Health University Institute, Montreal, QC, Canada
Department of Psychiatry, McGill University, Montreal, QC, Canada

3 Prevention and Early Intervention Program for Psychosis (PEPP), Douglas Mental Health University Institute, Montreal, QC, Canada 
examples of how the ACCESS OM project has implemented SDM processes, and discusses challenges encountered in this regard, with the aim of helping other projects and organizations implement SDM strategies.

\section{Key Points for Decision Makers}

ACCESS Open Minds has implemented SDM strategies to inform youth mental health service reform and research in Canada. The implementation of SDM strategies was facilitated by building relationships, sharing knowledge, and maintaining meaningful partnerships between all stakeholder groups.

Power dynamics, time constraints, project pace, and tokenism remain a challenge to SDM.

Lack of sustained project funding jeopardizes future activities.

\section{Introduction}

ACCESS Open Minds (ACCESS OM) is a patient-oriented research initiative that is transforming and evaluating youth mental health services at more than 16 diverse sites across Canada [1]. This transformation focuses on five core elements, namely early identification of mental health problems, rapid access to care, appropriate care, continuity of care, and youth and family/carer engagement [2]. Site teams are providing high-quality, timely, and easily accessible youth mental health services, and they are concurrently evaluating how this transformation is leading to better youth mental health outcomes [1].

Shared decision making (SDM) has been defined as a process by which health care providers and health care users collaborate and share knowledge with each other to come to decisions about the care provided [3, 4]. Making decisions about care based on mutually shared ideas can be considered inherently good, as the outcome will be the product of shared principles. Some authors note that SDM improves treatment processes and outcomes [5]. For example, studies have shown that the use of shared decision making in mental health care settings can improve patients' attitudes towards recovery [6] and, service providers' and patients' satisfaction with treatment decisions [7]. Although SDM concepts have been adopted in the field of mental health, literature describing and evaluating SDM strategies specific to this field remain relatively scarce [8-10]. However, there is widespread consensus that employing SDM strategies in youth mental health settings is critical to providing high quality, resilience-based, recovery-oriented youth mental health care $[11,12]$.
Funded under the Canadian Institutes of Health Research (CIHR)'s Strategy for Patient-Oriented Research (SPOR), the members of the ACCESS OM network recognized the importance of SDM from the outset, and therefore integrated it into the network's operations in accordance with the project's mandate for patient engagement. ACCESS OM defines 'patients' as youth aged 11-25 years, and 'patient partners' includes youth patient partners and, family and carers patient partners. 'Patient partners' contributed to the project based on lived mental health experience whereas 'patients' received care at an ACCESS OM site. 'Families and carers' include any significant and supportive person in a youth's life. Lived mental health experience is acknowledged and integrated as unique experiential knowledge and unique expertise to advise and to guide the project in collaboration with researchers, mental health clinicians, and decision makers.

Identified as an essential component to the network's activities, SDM strategies were woven into the project's governance structure, the design and execution of its mixed methods research and evaluation activities, and into the design of youth mental health services in diverse communities across Canada.

This paper shares insights from the co-design and implementation of SDM in knowledge translation, clinical, operational, and research strategies within the ACCESS OM network. The discussion explores operational and value-based challenges and provides practical recommendations to improve SDM in health care research and delivery. This article was written together by a team of youth and family partners, operational staff, and lead researchers, and so its publication serves in and of itself as an illustration of SDM between different stakeholders within the network. As ACCESS OM sites serve a diversity of communities - urban, rural, remote, Indigenous, Anglophone, Francophone, post-secondary, homeless youthin seven provinces and one territory, the insights provided here have widespread applicability for other contexts.

\section{Shared Decision Making (SDM) Practices and ACCESS OM's Knowledge Translation Strategy}

A great challenge within research is bridging the gap between generated knowledge and its implementation into practice, often described as the evidence-to-action gap. This gap can result in health care services that are inefficient and inappropriate [13], as they do not offer the level of innovation needed to respond to contemporary health problems. 
Knowledge translation (and knowledge mobilization, which is a closely linked concept) presents itself as a potential solution to this gap. Knowledge translation (KT) is the dissemination and application of knowledge, which can lead to the improvement of health care systems and better health outcomes [14]. Many funding agencies and research teams have integrated knowledge translation into their initiatives to help bridge the gap between research and practice, to support evidence-informed policies, and ultimately to improve care for all.

The ACCESS OM network has developed and adopted SDM strategies to support its knowledge translation objectives. Moreover, since the intersection between SDM and knowledge translation is an area of study that has not been given much attention, ACCESS OM is assessing the innovative ways in which SDM strategies might foster effective integrated knowledge translation (IKT) in a youth mental health research setting.

From the outset of the project, ACCESS OM adopted an IKT approach, in which knowledge users are included in all aspects of the project. A 'knowledge user' is any individual who will be able to use the knowledge that is produced by the project to inform any future decisions about health policy and practice. ACCESS OM chose an IKT approach as it can lead to more accurate findings and increase the use of these findings in policy and practice [14]. ACCESS OM knowledge users, also referred to as key stakeholders, are youth, family and carers, service providers, policy makers, funders, community partners, and researchers. Through implementing various SDM strategies, ACCESS OM has facilitated the development of partnerships between these different knowledge user groups.

One of the most important elements of IKT is that it allows for various forms of knowledge to co-exist in research projects [15]. Until recently, research projects often relied solely on scientific knowledge (acquired through research) to make recommendations. However, the use of IKT acknowledges that other forms of knowledge are just as valuable, important, and relevant as scientific knowledge. Through ACCESS OM's IKT strategy, three other types of knowledge are considered in decision-making processes: experiential knowledge (learned through experience), pragmatic knowledge (learned through action), and cultural knowledge (learned through being) [16]. ACCESS OM's scientific knowledge is composed of data gathered both qualitatively and quantitatively at the research sites and throughout the network. Experiential knowledge refers to the knowledge acquired by the project's stakeholders through their experience of mental health problems and services. Pragmatic knowledge refers to lessons learned by the project's stakeholders through the process of service transformation. Finally, cultural knowledge refers to the traditional knowledge held by all the individuals and groups from the different
ACCESS OM sites and communities. The acknowledgment of these various forms of knowledge allows key stakeholders to participate as true collaborators and co-creators alongside researchers and academics.

SDM has occurred in many areas of the ACCESS OM network: at the clinical level, in research, and in governance, all of which has promoted IKT throughout the project. At the clinical level, knowledge exchange has occurred between key stakeholders in the design of ACCESS OM services and care, and in the creation of KT products (e.g., flyers, posters, and webinars). SDM strategies were applied to ensure that all stakeholders were implicated in the development of the quantitative and qualitative research protocols, data analysis, and the development of KT products (e.g., publications, presentations, etc.). At the governance level, SDM has taken the form of knowledge exchange between key stakeholders on the Executive and Advisory Committees and the stakeholder councils.

\section{Embedding SDM in Governance Structures}

\subsection{Patient Partners as Members of Decision Making Councils and Committees}

Central to effective SDM is engaging patient partners as members in stakeholder committees and executive governance bodies at a governance level [17]. In the founding stages of ACCESS OM, it was determined that a redistribution of power among stakeholder groups was necessary to foster SDM with patient partners, researchers, clinicians, and decision makers. To do so, ACCESS OM invested in the development of separate and shared spaces between stakeholder groups to offer youth and family stakeholders resources to consult with each other, to move away from tokenism, and to consolidate strong messaging before contributing to larger multi-stakeholder discussions. It was therefore determined that ACCESS OM should have two patient councils: the ACCESS OM National Youth Council (NYC) and the ACCESS OM Family and Carers (AFC) Council. These councils would be represented by some of their members in the network's larger multi-stakeholder committees, namely the Executive Committee and the National Advisory Committee (see Fig. 1). These larger committees would act as the primary decision-making bodies for the overall initiative. The inclusion of and partnership with patient partners at an executive level enables professional relationship building and models the values of the ACCESS OM network. Relationship building is an essential element to youth and family engagement in research; these relationships create collaborative spaces between researchers and patient partners [18]. Youth patient partners and family/carer patient 
partners are voting members of the ACCESS OM Executive Committee in partnership with directors, clinicians, researchers, Indigenous community leaders, and site representatives (see Fig. 1). ACCESS OM welcomes all NYC and AFC Council members to attend Executive Committee meetings as observers (that is, as non-voting members) in an effort to promote transparency and knowledge sharing.

\subsection{Executive Committee Quorum Requirements}

As a further measure to underline the importance of patient perspectives within project decision-making processes, attaining quorum requires that both youth and family representatives are present before the committee can proceed with meetings. This requirement was instituted to support equal representation, and therefore equal opportunity, for knowledge exchange and deliberation of all stakeholder groups, a step that has been crucial in achieving SDM [11, 19].

\subsection{Organizational Veto Power}

Several strategies were implemented in the development of ACCESS OM's governance structure to facilitate the meaningful contribution of diverse stakeholder perspectives. These strategies included employing a consensus-based decision-making process, and reiterating the importance of patient perspectives and contributions by giving NYC and AFC Council members veto power. According to the consensus-based decision-making process adopted by the committee, a veto will halt the decision and return the matter back to the committee for discussion and further modification (as required) before being tested once again for consensus.
The allocation of veto power to youth and family/carer committee members is a strategy to underscore the value of and respect for youth and family/carer perspectives, which is crucial in moving away from paternalism and moving towards efficient SDM [3, 8, 9]. Through giving specific veto power to youth and family/carer committee members, the ACCESS OM governance structure is working to rebalance power among decision makers. ACCESS OM activities are guided by its fundamental voices: youth and family/carers in collaboration with clinicians, researchers, and decision makers. Furthermore, veto power shifts the pace of decision making by prolonging discussions and allowing time for in-depth discussion. Prolonged discussions create opportunity for capacity building. A key lesson has been that effective governance in a multi-stakeholder network that values patients' voices in decision making requires additional time and resources.

\subsection{SDM with Youth Patient Partners: the ACCESS OM National Youth Council}

The ACCESS OM National Youth Council (NYC) emerged as an opportunity for youth from communities across Canada to contribute their ideas and expertise to the project based on lived mental health experience. An annual operational budget is allocated to the NYC from the network grant funds for council activities and compensation. To increase transparency and share opportunities among council members, a peer nomination system is used to engage new members. Communication between these members occurs through monthly meetings and a private Facebook forum. SDM between youth council members helps to identify strategic priorities for the NYC such as membership, knowledge

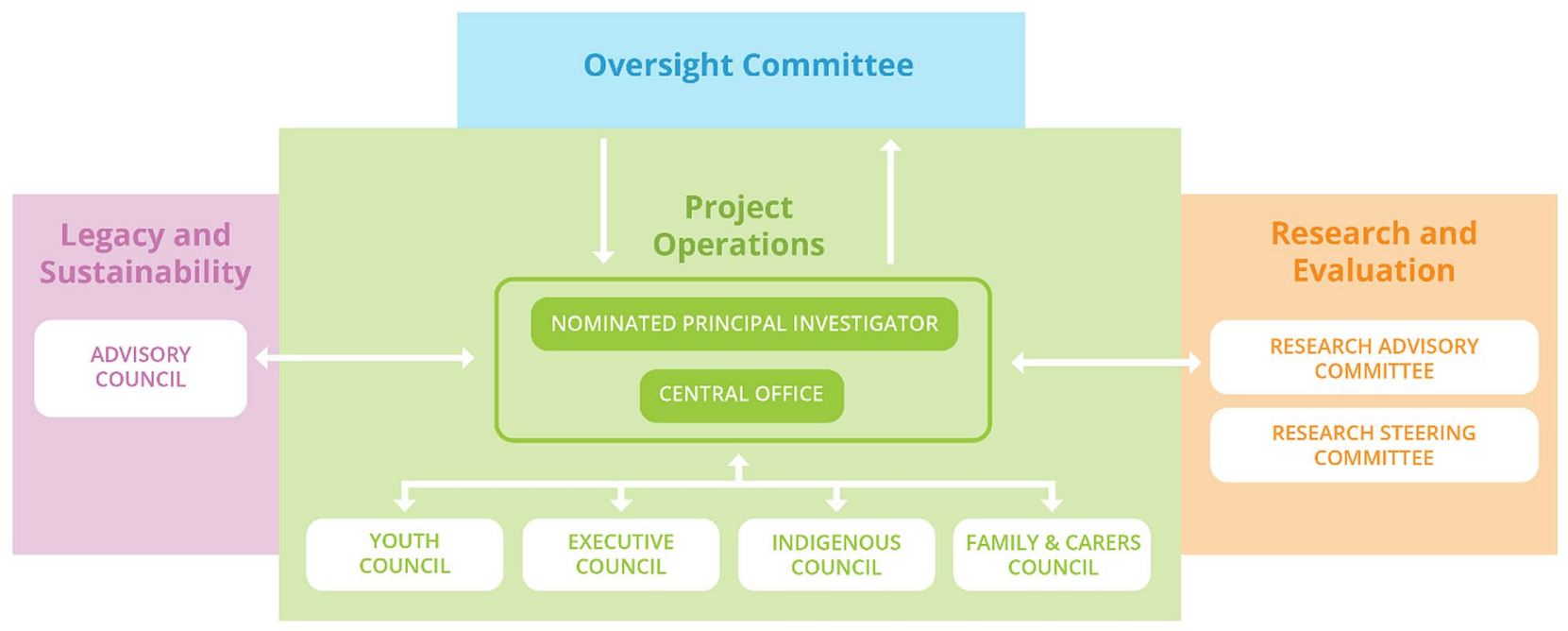

Fig. 1 The ACCESS OM governance structure: the network's councils and committees and their areas of governance 
translation, training, communications, etc. The NYC is cofacilitated by two elected co-leads and the ACCESS OM Stakeholder Engagement Coordinator (an operational staff member of the ACCESS OM central office) and the coleads represent the NYC on the ACCESS OM Executive Committee.

\subsection{SDM with Family and Carer Patient Partners: the ACCESS OM Family and Carers Council}

ACCESS OM recognizes the importance of incorporating knowledge stemming from lived experience of families and carers. Family members and carers with experience in supporting a loved one with a youth mental health concern have vital knowledge that can contribute to better outcomes for youth requiring mental health support [20, 21]. As such, ACCESS OM allocated significant resources to create and maintain a national community of practice for families and carers. This innovative space has fostered knowledge mobilization about family engagement and family peer support as part of best practices in youth mental health services. Across the network, AFC Council members are integrated into all aspects of the ACCESS OM initiative, including the delivery and design of clinical services, research activities, and on governing bodies.

AFC Council members include individuals from communities across Canada with lived experience of supporting a family member with mental health problems, as well as family peer support providers from ACCESS OM sites who are dedicated to contributing their experience to improve youth mental health services in Canada. The AFC Council has created the opportunity for meaningful spaces for family and carers to share their perspectives and contribute to decision making regarding the role of family and carers in youth mental health care. The AFC Council strives to counter feelings of exclusion that are often experienced by families and carers [22] by connecting family members with one another. The Council also shares insights into youth mental health care based on members' experiential knowledge of caregiving. This knowledge is shared from a culturally and geographically diverse membership of people from rural, remote, urban, and Indigenous communities across Canada.

The AFC Council is guided by their terms of reference, annual budget, and work plan. The Council's Chair is a member of the ACCESS OM Executive Committee and the Research Advisory Committee, and works alongside the project's decision makers and community stakeholders. Governance of the AFC is an integrated process that involves all members of the council. Members are invited to provide ideas, concepts, and values that inform how the council is governed. A seminal governance document was created over many months through which revisions were made in order to best reflect the voices of its members. Once drafted, each member had an opportunity to review and vote on the adoption of the document. A majority vote was needed for this document to take effect. Over the lifespan of the ACCESS Open Minds project, revisions were made to reflect the ongoing growth and development of the council. Each time, members were given the chance to review and vote on the document. The AFC has also been influencing site activities; for instance, family peer support grew to become a pillar of service at several sites because of the work of AFC Council members.

As patient partners working towards a common goal, members of the NYC and the AFC Council established an alliance to jointly create initiatives for both youth and families. For example, the two councils collaborated with the research team to design a photovoice project; the joint team contributed towards writing the proposal, developing a budget, designing a training program, recruiting stakeholder participants, and conducting analysis. This project, which currently includes participants from 10 sites, has managed to engage over 100 stakeholders, including youth, family and carers, researchers, and members of management.

\section{SDM in Service Contexts}

At its core, all ACCESS OM activities focus on transforming youth mental health services to improve mental health outcomes for youth and their families. SDM between youth (the patient), their family/carer, and the clinician/worker providing care is pivotal to this transformation and to improving treatment outcomes [5]. Numerous SDM strategies were implemented across ACCESS OM sites, described in the following sections.

\subsection{Development and Adherence to Core Values}

To guide this shift in mental health care delivery, clinicians, youth, and family/carers initiated an internal working group mandated to develop a set of core values to guide service providers at ACCESS OM sites. The purpose of this working group was to define shared values considered fundamental to quality youth mental health services. The literature suggests that SDM can lead to a better alignment of patients' needs and providers' perspectives [3]. ACCESS OM works to promote sustained SDM in care delivery by adhering to and integrating values that were developed with patient partners. These values elicit a shift in practices [23] specific to youth mental health settings. In clinical settings, SDM is often perceived as a challenge, as decisions are often based solely on medical and psychiatric expertise [23]. Adherence to mutually agreed-upon core values challenges this reliance and helps clinicians strive to include patient perspectives in clinical decision making. ACCESS OM sites visibly display 
these core values in their accessible youth spaces to inform and remind youth and their family/carers-as well as the clinicians providing care - of the importance of adhering to these values. These values ultimately serve as guidelines for SDM in clinical care. Examples of the core values featured on this list include involvement of youth and families/carers in assessment and decision making about treatment, and valuing of diversity and strengths.

\subsection{Multi-Stakeholder Hiring Panels}

National Youth Council members called for a radical shift in hiring practices by tabling an Executive Committee motion to include youth and family stakeholders in the hiring of all ACCESS OM staff, both at sites and at the central office. Executive Committee members welcomed this SDM initiative as a strategy to hire clinicians and researchers who would best work with youth as valued partners. Leadership at the ACCESS OM sites and central office partnered with youth and family/carers to hire many staff members using this method, including research assistants, site clinicians, operational staff, and the network's Director of Services and Engagement. Patient and family partners contributed their expertise by reviewing applications, conducting interviews with the hiring panel, and providing their recommendations. This SDM strategy was initiated to respond to the discrepancy between patient needs and the actual care that the system provides. Literature suggests that effective SDM could be facilitated by staff members that have certain personality factors and relational factors such as openness and trust [24, 25]. ACCESS OM aimed to create a workforce reflective of patient needs and preferences by incorporating SDM into hiring processes with youth and family stakeholders.

Following the implementation of multi-stakeholder hiring panels, an NYC member proposed a KT project that invited local stakeholders who participated in the hiring panel to share insights on their experience. These learnings were disseminated through a four-part video series available on YouTube [26], and shared at multiple conferences. Quotations from the shared experience gleaned through these video interviews were collated into four themes: (i) inspiration, (ii) experience, (iii) challenges, and (iv) lessons learned. Figure 2 illustrates a summary of the experience of hiring panel members.

\subsection{Training}

Within the ACCESS OM network, a training program was created and delivered that includes learning objectives related to transforming systems of care, integrating standardized evaluation tools into clinical care, and youth and family engagement within service design and in the delivery of care, among other topics. Patient partners contributed to the development and delivery of training. Youth assisted in writing clinical scenarios to prompt dialog about best practices in SDM in youth mental health clinical settings. NYC members were also asked to illustrate their answers to the following question: "What would you like a clinician to keep in mind when they are providing care to youth?" These stories generated discussions with the clinical teams around barriers to SDM, and factors facilitating SDM.

\subsection{ACCESS OM Youth Spaces}

At ACCESS OM sites, local stakeholders, including youth patient partners, came together to assist in determining the design of the site's youth space as well as the programming and services to be offered. SDM principles were operationalized in multiple ways through this process: setting parameters around which decisions youth could (e.g., interior design) and could not (e.g., funds available) influence, negotiating differences in opinions and priorities, and learning about new things (e.g., real estate rules, patient safety and space, etc.) to enable SDM.

SDM resulted in youth-friendly spaces that offer activities responding to the specific needs and preferences of youth in each community. Activities include on-site mental health services, gaming and movie nights, music programming, cooking classes, sports and traditional programming (such as drum making and on-the-land activities). The act of codesigning a space also fostered a sense of shared ownership among a community's youth, and ensured that spaces were culturally relevant to the youth being served (e.g., through the artwork and posters that decorate the space and the tools and materials available to youth and staff to perform the site's activities).

\section{Shared Decision Making in Research}

\subsection{Mixed Methods Research Strategy and Consent Forms}

The NYC and AFC Council members contributed to the development of the ACCESS OM qualitative and quantitative research strategies, providing input on the instruments and measures to be used, as well as what information would be important to collect [27]. A working group with representatives from these two councils and each participating site was created to provide feedback on key outcome domains and measures that should form part of the ACCESS OM's quantitative assessment protocol. Their inclusion highlighted important criteria for choice of assessments tools (e.g., short tools with youth-friendly language, domains that go beyond symptoms, etc.) and item response options-for example, the demographic questionnaire has an expanded 


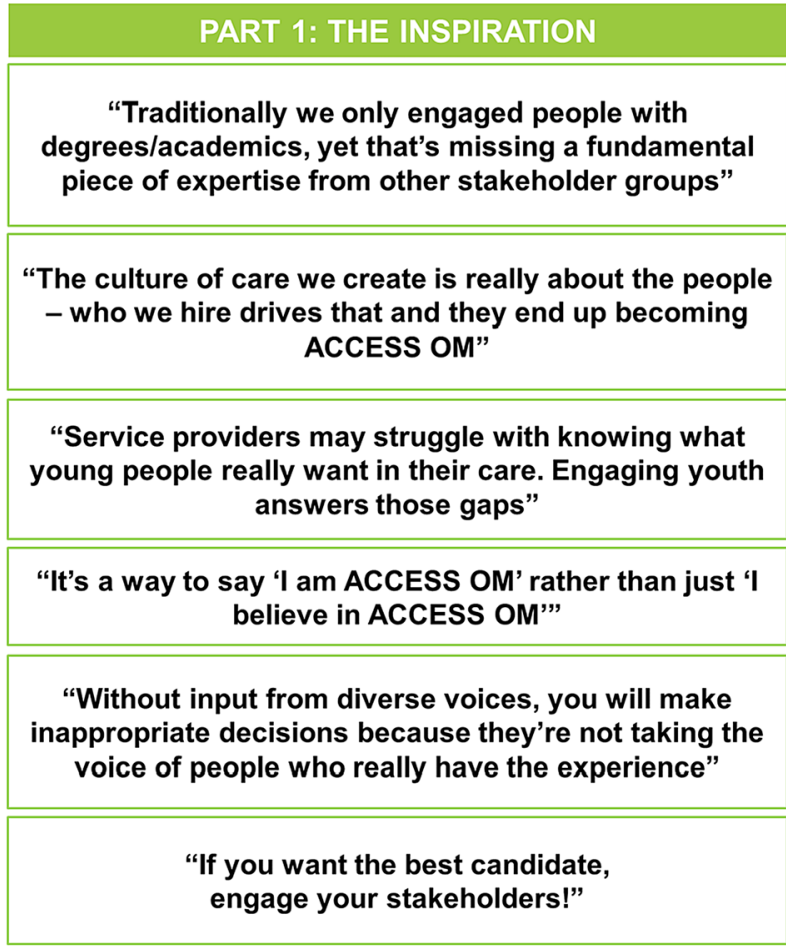

\begin{tabular}{|c|}
\hline PART 3: THE CHALLENGES \\
\hline $\begin{array}{c}\text { "The commitment was in values. If there was } \\
\text { hesitation it was how to make it possible for now, } \\
\text { and for in the future" }\end{array}$ \\
\hline "Making sure their voices are heard" \\
\hline $\begin{array}{c}\text { "Group think was a genuine concern of mine. [...] } \\
\text { because of the power dynamic that } \\
\text { traditionally exist in the system" }\end{array}$ \\
\hline $\begin{array}{c}\text { "There may be people or youth who would like to } \\
\text { participate but they don't know how to do it. } \\
\text { Do not be afraid. Go for it! } \\
\text { There is always someone who will support you" }\end{array}$ \\
\hline $\begin{array}{c}\text { "Give the panelists some [organizational] history so that } \\
\text { they have awareness of the project [...] } \\
\text { and also talking to them about } \\
\text { their hopes, dreams and wishes for this project" }\end{array}$ \\
\hline $\begin{array}{l}\text { "To just speak simply about the things we need to be } \\
\text { ready for" }\end{array}$ \\
\hline $\begin{array}{c}\text { "Oftentimes we make things complicated when really } \\
\text { they don't have to be" }\end{array}$ \\
\hline
\end{tabular}

\section{PART 2: THE EXPERIENCE}

"We did not make this optional; it was mandatory. Rules are what needed to break other rules"

"I've never done this before! It's so humbling to offer my experience to serve my community"

"You may be interviewing one candidate, but they are perceived by an array of stakeholder perspectives. That is the magic of diversity"

"You pick up information not only on how they answered your question, but also how they interacted with the youth, family, and others"

"Something l've never thought of - which a youth came up with - was role plays during the interview. You aren't asking the standard interview questions"

"Having been an interviewee, the experience I had

reminds me of why l'm here: to continue engaging diverse stakeholders in my day to day job"

"How come we didn't think of this earlier??"

\section{PART 4: LESSONS LEARNED}

"You're going to get the best of the best"

"We are covering a generation gap"

"There has to be buy in from senior leadership"

"It does take more time"

"The responsibility of the decision making is not only on me. It's nice to know that many people are participating in this decision"

"It was eye opening to learn about the point of view of other employees [...] I think it's important for everyone to know other people's point of views"

"We too can have prejudices, mental representations that we have made of young people. The project invites us to put that aside and go discover someone else"

"We're only at the beginning of this journey [...] we also have a lot more to learn"

Fig. 2 Youth-led knowledge translation project on multi-stakeholder hiring panels

set of options for sexual orientation and gender identity that were provided by youth advisors. Informal feedback from site service providers has indicated that young service users at all sites have appreciated the range of options provided for these two questions and seen this as an indicator of ACCESS OM sites being "safe spaces".

A notable SDM activity involved youth and family/carers in working groups and consultations to influence the 
redesign of research consent forms. Patient partners had initially reported that the original youth consent forms were too lengthy and difficult to understand. In response, a committee including patient partners was established to review the forms and isolate the fundamental components to co-create the Care Consent Form. This form, reduced from its original eleven pages to two pages, is written in youth-friendly language to support and enhance SDM practices in research recruitment. This change represented an innovation in health services research. While the local Research Ethics Board was initially reluctant to accept the changes, the new form was ultimately approved as it prioritized the youth voiceand ultimately youth engagement in care and research-all the while maintaining the fundamental elements of the original consent form.

\subsection{Research Advisory Group}

The ACCESS OM Research Advisory Group meets quarterly to provide oversight on the research and evaluation activities of the network. A minimum of one NYC member and one AFC Council member is required to reach quorum.

\subsection{Publications Committee}

The ACCESS OM Publications Committee provides oversight of conference abstracts, manuscript submissions, and contributions to various publications. Representatives from both the NYC and AFC Council are members of this committee. They contribute to the review of submissions brought forward by the ACCESS OM network, and they provide comments for consideration, such as journal submission choice, vocabulary choices, and topic relevance to stakeholders.

\section{SDM and Capacity Building}

\subsection{Knowledge Translation}

ACCESS OM's knowledge translation (KT) strategy involves the dissemination of various types of knowledge using a variety of media. SDM was integrated into this phase of the KT strategy by inviting NYC and AFC Council members to lead the development of multiple digital, social media, and paper resources. These resources included onepage documents outlining topics relevant to youth and family patient partners, webinars, and social media posts using infographics and videos.

Knowledge sharing at academic and community conferences has played a pivotal role in the knowledge dissemination strategy of the ACCESS OM network. Youth and family/carers council members have co-developed presentations and delivered them together at conferences. Notably, patient partners presented at the 2019 International Shared Decision Making Conference in Québec City, the 2018 Canadian Mental Health Association Annual Conference, as well as at both the 2017 and 2019 conferences of the International Association for Youth Mental Health (in Ireland and Australia, respectively). Allocating budget to council activities has empowered patient partners to pursue and contribute to knowledge translation throughout the network and beyond.

\subsection{Project Branding, Website, and Social Media}

The name of the project, "ACCESS Open Minds" is an acronym where ACCESS stands for Adolescent/young adult Connections to Community-driven, Early, Strengths-based and Stigma-free services. Coming up with this acronym that reflected shared values and aspirations was one of the first activities the network's multiple stakeholders engaged in, using SDM principles. Subsequently, the project's logo and brand colors were co-created and co-designed with patient partners. These SDM strategies have resulted in a brand that is reflective of patients' preferences.

\subsection{Capacity Building, Scaling Up, and Sustainability}

Patient partners have participated in critical discussions regarding how to secure financial sustainability and policy buy-in for the continuation of the ACCESS OM network's initiatives beyond the original 5-year mandate; these conversations have been fundamental to the perpetuation of this pan-Canadian research and evaluation network. Various SDM strategies have been implemented to inform sustainability and scale-up efforts. To promote shared discussion and decision making, sustainability efforts have been presented to working groups, committees, and councils that comprise multiple stakeholders. Furthermore, individual interviews were also conducted with youth and family council members to discuss sustainability and a vision for scaling up the ACCESS OM framework. Moreover, marketing and communications strategies that aim to raise awareness about the project and secure further funding have been informed by patient partners.

Knowledge translation activities include patient partners. For example, patient partners wrote commentaries about the network's transformation model, published in an ACCESS OM-specific supplement in Early Intervention in Psychiatry. Conference presentations and panel discussions regularly include patient partners (International Association of Youth Mental Health Conference, 2017, Dublin, Ireland; International Association of Youth Mental Health Conference, 
2019, Brisbane, Australia; Canadian Mental Health Association conference, 2019, Toronto; Canadian Mental Health Association conference, 2018, Montreal; National Conference on Peer Support, 2018, Calgary; SPOR Summit, 2018, Ottawa).

ACCESS OM network members across Canada have succeeded in mobilizing resources in their communities and have been transforming their youth mental health services. An important achievement of the project has been the basing of its transformative endeavors in core principles (such as SDM), objectives, and protocols that have been contextualized to the realities of diverse sites. We have already described these core principles and the successful contextualization of these principles towards the creation of transformed youth mental health services in 14 contexts in our previous publications $[1,2,27]$. In the future, we look to publish results regarding impacts of transformed services on reach, wait times, outcomes, and service users' satisfaction with services, and on the processes underpinning youth mental health services transformation and the factors fostering and hindering such transformation. Results are expected to generate meaningful directions for continuing youth mental health research, policy, and service efforts.

The ACCESS OM network has invested in knowledge sharing among stakeholders from various provincial and territorial jurisdictions. Patient partners connect with each other and empower one another. Knowledge sharing between patient partners from diverse communities has contributed to the implementation of SDM practices within governance structures, clinical, and research settings. Insights from youth and family patient partners describe how vital the ACCESS OM network has been to the fulfillment of their role within ACCESS OM and within their own community. Just as researchers and clinicians have professional associations where they can learn and grow, patient partners also need dedicated spaces to network, strategize, and build capacity.

ACCESS OM has empowered patient partners to speak from their lived experience to inform decisions on youth mental health system reform in Canada. SDM within this project has informed service delivery and research activities. Together, the ACCESS OM network members are actively seeking funding and policy buy-into sustain promising practices in youth mental health care across Canada.

\section{Challenges and Lessons Learned}

A summary of strategies used by ACCESS OM to implement and sustain SDM principles is presented in Table 1 . Implementing effective SDM strategies at all levels of
ACCESS OM has not been without challenges, however. In the following sections we outline the main challenges faced, and some strategies to address each challenge.

\subsection{Time- and Pace-Related Constraints}

Challenge It is vital to SDM to invest in creating working environments and relationships where all stakeholders can be heard and empowered.

Time constraints and the associated project pace have been noted as barriers to SDM in clinical settings [28]. These factors have also presented as threats to SDM within the ACCESS OM network. Creating space and investing the time required to maintain professional relationships is a challenge in a pan-Canadian project. Coordination is required to overcome significant geographical distances and multiple time zones in order to ensure that stakeholders' participation is accessible. Even in the same time zone, operating within standard 9:00 am to 5:00 pm business hours can represent a significant barrier to stakeholder engagement, and consequently to implementing SDM with youth and family patient partners. Family engagement proved at times difficult to maintain across the network, with barriers for involvement stemming from family obligations, work schedules, and the perception of a lack of meaningful engagement.

Recommendations Recommendations include setting reoccurring meeting times well in advance, incorporating quorums and veto power at meetings, and allowing staff members to work flexible hours. Youth specifically requested alternating meeting times between weekday daytime hours and weekends to promote attendance. ACCESS OM network members have demonstrated the willingness to adapt timelines by adopting a consensus decision-making framework into governing bodies.

We also recommend the creation of multi-stakeholder working groups to draft guidelines on SDM values and communication guidelines. For SDM to occur, all members need to agree on timelines and be informed about expectations in order to respect their own self-care and wellness while meeting project deadlines. Respecting a deadline should not trump someone's wellbeing. Developing trusting collegial relationships creates space for difficult discussions about potentially sensitive topics, like deadlines, support, and wellness.

\subsection{Power Dynamics and Tokenism}

Challenge Despite a commitment to SDM, multiple factors can impede SDM by reinforcing traditional hierarchies and power structures. In the case of ACCESS OM, this challenge was particularly salient early in the project and served 


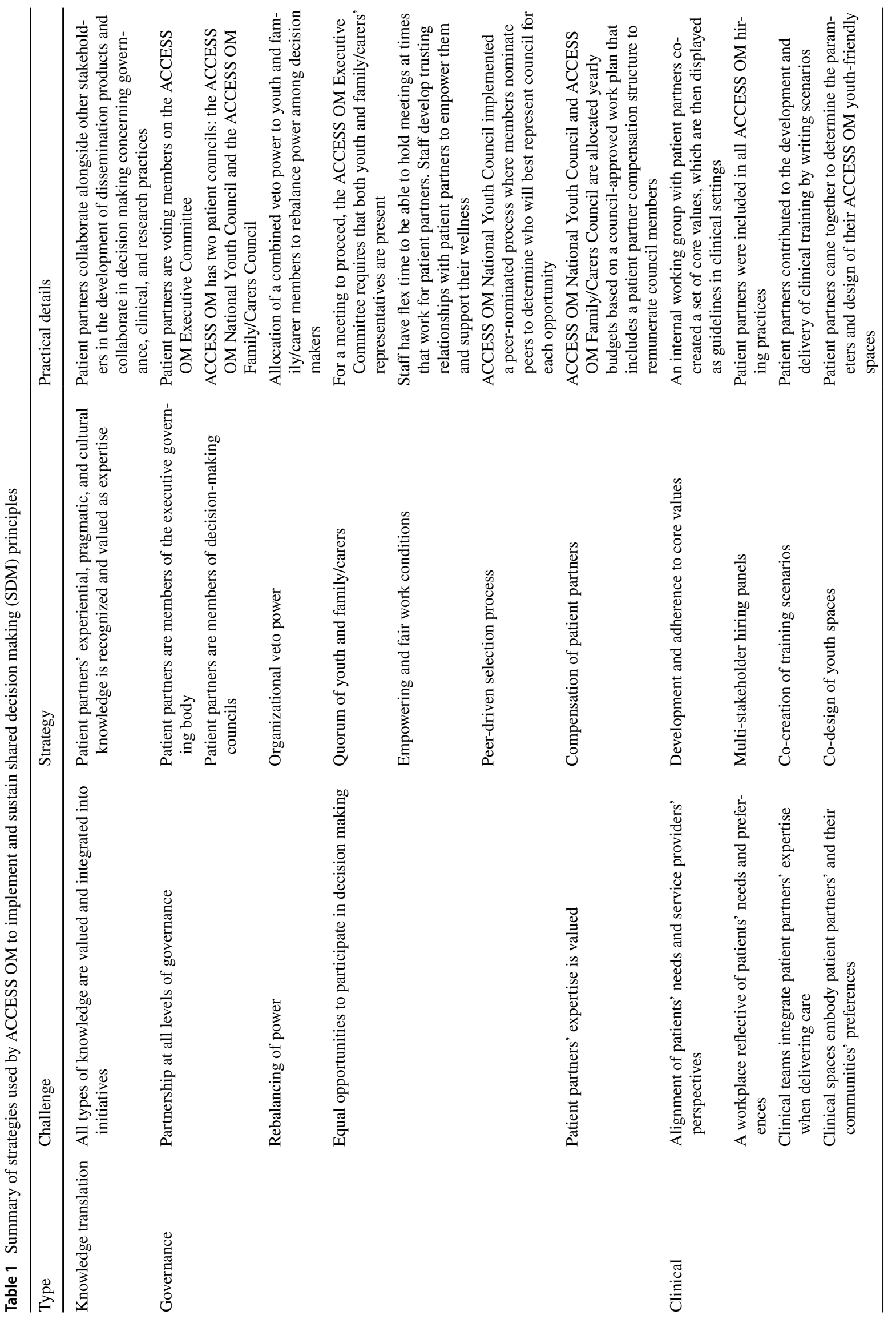


as a continued opportunity for reflection and improvement. The ability to understand and implement knowledge can be hindered by hesitation to change practices and develop new policies. Certain practices can hinder engagement and SDM, even when these values are held in high regard by the project. A major challenge and threat to patient involvement in decision making is the administrative structure and prevailing culture in parent institutions where the service or the network administration may be situated. Other examples within the ACCESS OM network include dedicating a limited amount of time and resources to patient engagement, or situating the decision-making process in an intimidating setting (e.g., a room full of researchers who are considered to be experts, alongside one or two patient partners).

Similarly, tokenism is a threat to SDM. Tokenism occurs when patient partners are not given an opportunity to be heard and involved in the decision-making process, and can lead to inauthentic engagement, especially in the context of youth patient partners [29]. Within ACCESS OM, the concept of tokenism has been extended to include the overrepresentation of one stakeholder voice. Tokenism negatively impacts trust and relationships with the stakeholder population [30].

Recommendations Shifting power dynamics requires a strong, consistent, and ethical leadership team that adheres to and promotes SDM values and activities. Understanding what and whose knowledge is considered important in the research setting is crucial. All stakeholders need to cooperate and invest significant effort to create change. Investments in training and change management practices need to be made. For instance, a Theory of Change was developed within the ACCESS OM network to inform strategic planning. Staff need to value patient partners' contributions to SDM practices. Furthermore, additional patient partners must be integrated into teams. Patient partners are needed in various forums to ensure that their voice is heard throughout all processes.

Creating an equitable space for patient partners is an important aspect of SDM in research. Compensating patient partners for their time and unique expertise plays a crucial role in the acknowledgement of this important partnership [31]. In doing so, research projects promote the value of patient expertise, create fairness among those who are doing the same kinds of work, generate opportunities for documenting patient partner contributions, and acquire a better understanding of the level of participation throughout the process [32]. Clear expectations around how patient partners should be compensated must be established from the outset. For example, the AFC Council and the NYC are each allocated an annual budget based on their council's annual work plan. The AFC Council work plan reflects the council's mission and objectives for family engagement and family peer support in ACCESS 
OM. The approved work plans include a structure outlining remuneration for patient partner compensation. Thus, the ACCESS OM network is deliberately engaging patient partners in a meaningful way for sharing their expertise, as well as showing value for the time and effort they dedicate to the project, all of which is consistent with the growing consensus regarding the value of compensating patient partners in health research [18].

In an effort to counter tokenism, the NYC implemented a nomination process in decision-making situations. Members would nominate a peer, or self-nominate, to determine who would best represent the Council, an approach that led to a peer-driven selection process for representation within ACCESS OM initiatives. Additionally, the NYC and other ACCESS OM governing bodies are intentionally composed of leaders from the local site communities, ensuring that cultural and experiential knowledge is incorporated into research and service design through SDM. This approach has created opportunities for increased membership, and has empowered under-represented voices within the project. While this peer nomination system cannot address the depth of systemic discrimination or under-representation of minority groups, it does increase transparency in decision-making processes, and empowers members to recognize the qualities of their teammates. NYC members purposefully removed application forms or membership criteria to remove barriers and create an accessible membership process for all youth, including youth from under-represented groups. For instance, youth from any community across Canada are welcome to join the NYC so long as they are inspired by the council's mandate and speak from the perspective of someone with lived experience of mental health. As such, membership to the NYC includes young people from a number of different cultural communities, socio-economic backgrounds, linguistic groups, and members of the LGBTQ2 + community. Peer training on anti-oppressive practices and privilege was offered to NYC members to increase awareness of privilege and oppressive structures. Education, training, and opportunity for advancement is an attempt to increase participation and leadership from all members. Support is provided through remuneration, translation, one-on-one discussion, group discussions, and testimonials about culture, diversity, racism, and other topics that youth would like to address. ACCESS OM experienced a challenge in engaging youth from some rural and northern communities, given that their participation requires reliable access to the Internet. As a result, it was deemed important to host in-person meetings twice a year, where travel and accommodation expenses, as well as remuneration for time, is included in the council's budget to ensure this meeting's accessibility to all members. Project directors are invited to this meeting as well to create an opportunity for information sharing.

\section{Conclusion}

ACCESS OM acknowledges and values the lived experience of patients and, families and carers as unique expertise. Governance structures within the ACCESS OM network have been established to promote stakeholder engagement in knowledge translation activities, in the design of youth mental health services, in research and evaluation development, implementation, and analysis. SDM strategies foster dialog and partnerships among stakeholders (e.g., youth, family members/carers, clinicians, researchers, and policy makers) by acknowledging that diverse forms of knowledge (specifically experiential, cultural, clinical, and scientific knowledge) can lead to better health and improved social and economic outcomes for patients and communities. Challenges to SDM include power dynamics, time constraints, project pace, and tokenism.

The strength of ACCESS OM is its pan-Canadian network: youth, family, carers, clinicians, researchers, and decision makers are all devoted to transforming youth mental health services in their communities and across Canada. Together, the ACCESS OM network strives to identify strategies to continuously improve and evaluate SDM practices within diverse youth mental health settings. Further, while previous SDM work in mental health has primarily focused on the individual encounter between patient and clinician, we have substantially extended SDM principles to other domains such as service design, knowledge translation, training, capacity building, and network governance. The journey taken by this network has generated insights into various innovative SDM practices and strategies that may be of value in other health care contexts. While these strategies have not been formally evaluated, we believe that they serve as inspiration for other projects, including those that seek to systematically implement and evaluate SDM practices.

Acknowledgements ACCESS Open Minds is a Strategy for PatientOriented Research (SPOR) network funded by the Canadian Institutes of Health Research and the Graham Boeckh Foundation. We also acknowledge the valuable contributions of the many additional ACCESS Open Minds Network members, including at its 16 sites, who contribute to this project and whose extensive efforts have contributed to its quality and spirit. The network includes a vibrant National Youth Council, a Family and Carers Council, an Indigenous Council and a central office in Montreal. The corresponding author can be contacted for a list of all network members. We would like to thank Margot Nossal for her assistance with reading and editing the manuscript. We would like to thank Catherine Lau for her assistance with designing tables and figures. 


\section{Declarations}

Funding SPOR network jointly funded by Canadian Institutes of Health Research (CIHR) and the Graham Boeckh Foundation.

Conflict of interest The authors have no conflict of interest to report in relation to this report.

Author Contributions Design and conception: CM, CG, JT, YP, SJ, AM, SI; First draft: CM, CG, JT, YP, SJ. All authors revised critically and approved the final version for publication, and meet the following four criteria for authorship recommended by International Committee of Medical Journal Editors (ICMJE): (1) substantial contributions to the conception and design of the work; (2) drafting the work or revising it critically for important intellectual content; (3) final approval of the version to be published; and (4) agreement to be accountable for all aspects of the work in ensuring that questions related to the accuracy or integrity of any part of the work are appropriately investigated and resolved.

Open Access This article is licensed under a Creative Commons Attribution-NonCommercial 4.0 International License, which permits any non-commercial use, sharing, adaptation, distribution and reproduction in any medium or format, as long as you give appropriate credit to the original author(s) and the source, provide a link to the Creative Commons licence, and indicate if changes were made. The images or other third party material in this article are included in the article's Creative Commons licence, unless indicated otherwise in a credit line to the material. If material is not included in the article's Creative Commons licence and your intended use is not permitted by statutory regulation or exceeds the permitted use, you will need to obtain permission directly from the copyright holder. To view a copy of this licence, visit http://creativecommons.org/licenses/by-nc/4.0/.

\section{References}

1. Iyer SN, Boksa P, Joober R. How youth mental healthcare is being transformed in diverse settings across Canada: reflections on the experience of the ACCESS Open Minds network. Early Interv Psychiatry. 2019;13:8-11.

2. Malla A, Iyer S, Shah J, Joober R, Boksa P, Lal S, Hutt-MacLeod D. Canadian response to need for transformation of youth mental health services: ACCESS open minds (esprits ouverts). Early Interv Psychiatry. 2019;13(3):697-706.

3. Charles C, Gafni A, Whelan T. Shared decision-making in the medical encounter: what does it mean?(or it takes at least two to tango). Soc Sci Med. 1997;44(5):681-92.

4. Montori VM, Gafni A, Charles C. A shared treatment decision-making approach between patients with chronic conditions and their clinicians: the case of diabetes. Health Expect. 2006;9(1):25-36.

5. Langer DA, Jensen-Doss A. Shared decision-making in youth mental health care: using the evidence to plan treatments collaboratively. J Clin Child Adolesc Psychol. 2018;47(5):821-31.

6. Dixon LB, Glynn SM, Cohen AN, Drapalski AL, Medoff D, Fang LJ, Gioia D. Outcomes of a brief program, REORDER, to promote consumer recovery and family involvement in care. Psychiatr Serv. 2014;65(1):116-20.

7. Loh A, Simon D, Wills CE, Kriston L, Niebling W, Härter M. The effects of a shared decision-making intervention in primary care of depression: a cluster-randomized controlled trial. Patient Educ Couns. 2007;67(3):324-32.
8. Adams JR, Drake RE. Shared decision-making and evidencebased practice. Community Ment Health J. 2006;42(1):87-105.

9. Duncan E, Best C, Hagen S. Shared decision making interventions for people with mental health conditions (protocol). The Cochrance Collaboration (2009). https://www.ispraisrael.org.il/ Items/01837/CD007297/5B1/5D.Pdf. Accessed 5 Mar 2014.

10. Duncan E, Best C, Hagen S. Shared decision making interventions for people with mental health conditions. Cochrane Database Syst Rev. 2010;1:CD007297.

11. Grim K, Rosenberg D, Svedberg P, Schön U. Shared decisionmaking in mental health care-a user perspective on decisional needs in community-based services. Int J Qual Stud Health WellBeing. 2016;11(1):30563.

12. Simmons MB, Batchelor S, Dimopoulos-Bick T, Howe D. The choice project: peer workers promoting shared decision making at a youth mental health service. Psychiatr Serv. 2017;68(8):764-70.

13. Jull J, Giles A, Graham ID. Community-based participatory research and integrated knowledge translation: advancing the cocreation of knowledge. Implement Sci. 2017;12(1):150.

14. Canadian Institutes of Health Research (CIHR). About us: knowledge translation (2016). http://www.cihr-irsc.gc.ca/e/29418 .html\#2. Accessed 28 Jan 2020.

15. Jull J, Hizaka A, Sheppard A, Kewayosh A, Doering P, MacLeod L, Team The Inuit, Interpreter Medical. An integrated knowledge translation approach to develop a shared decision-making strategy for use by inuit in cancer care: a qualitative study. Curr Oncol. 2019;26(3):192.

16. Knowledge Exchange Centre of the Mental Health Commission of Canada, Bilsker D, Goldner EM (2012) A practical guide to knowledge translation in health care. https://www.mentalheal thcommission.ca/sites/default/files/2016-06/innovation_to_imple mentation_guide_eng_2016_0.pdf. Accessed 28 Jan 2020.

17. Ramey HL, Lawford HL, Vachon W. Youth-adult partnerships in work with youth: an overview. J Youth Dev. 2017;12(4):38-60.

18. Woodgate RL, Zurba M, Tennent P. Advancing patient engagement: youth and family participation in health research communities of practice. Res Involv Engagem. 2018;4(1):9.

19. Elwyn G, Frosch D, Thomson R, Joseph-Williams N, Lloyd A, Kinnersley P, Rollnick S. Shared decision making: a model for clinical practice. J Gen Intern Med. 2012;27(10):1361-7.

20. Chovil N, Panagiotopoulos C. Engaging families in research to determine health literacy needs related to the use of second-generation antipsychotics in children and adolescents. J Can Acad Child Adolesc Psychiatry J De l'Academie Canadienne De Psychiatrie De l'Enfant Et De l'Adolescent. 2010;19(3):201-8.

21. MacKean G, Spragins W, L'Heureux L, Popp J, Wilkes C, Lipton $\mathrm{H}$. Advancing family-centred care in child and adolescent mental health: a critical review of the literature. Healthc Q. (Toronto Ont.). 2012;15(Spec No 4):64-75. https://doi.org/10.12927/ hcq.2013.22939.

22. Duchnowski AJ, Kutash K. Family-driven care. Tampa: University of South Florida; 2007.

23. Prilleltensky I, Peirson L, Gould J, Nelson G. Planning mental health services for children and youth: part I-a value-based approach. Eval Progr Plan. 1997;20(2):163-72.

24. Street RL Jr, Makoul G, Arora NK, Epstein RM. How does communication heal? Pathways linking clinician-patient communication to health outcomes. Patient Educ Couns. 2009;74(3):295-301.

25. Epstein RM, Street RL Jr. Shared mind: communication, decision making, and autonomy in serious illness. Ann Fam Med. 2011;9(5):454-61. https://doi.org/10.1370/afm.1301.

26. ACCESS Open Minds (2018) ACCESS open minds-hiring process: a four part video series. https://youtu.be/PvK0DDg_uqI. Accessed 8 Apr 2020.

27. Iyer SN, Shah J, Boksa P, et al. A minimum evaluation protocol and stepped-wedge cluster randomized trial of ACCESS Open 
Minds, a large Canadian youth mental health services transformation project. BMC Psychiatry. 2019;19:273. https://doi. org/10.1186/s12888-019-2232-2.

28. Chong WW, Aslani P, Chen TF. Shared decision-making and interprofessional collaboration in mental healthcare: A qualitative study exploring perceptions of barriers and facilitators. J Interprof Care. 2013;27(5):373-9.

29. Hawke LD, Relihan J, Miller J, McCann E, Rong J, Darnay K, Henderson JL. Engaging youth in research planning, design and execution: practical recommendations for researchers. Health Expect. 2018;21(6):944-9.

30. Hawke LD, Mehra K, Settipani C, Relihan J, Darnay K, Chaim $\mathrm{G}$, Henderson J. What makes mental health and substance use services youth friendly? A scoping review of literature. BMC Health Serv Res. 2019;19(1):257.

31. SPOR Networks in Chronic Diseases and the PICHI Network. Recommendations on patient engagement compensation (2018). https://diabetesaction.ca/wp-content/uploads/2018/07/TASKFORCE-IN-PATIENT-ENGAGEMENT-COMPENSATIONREPORT_FINAL-1.pdf. Accessed 9 Apr 2020.

32. Canadian Institutes of Health Research (CIHR). Considerations when paying patient partners in research (2019). https://cihr-irsc. gc.ca/e/51466.html. Accessed 9 Apr 2020. 